\title{
Rechtsgeschichte
}

www.rg.mpg.de

http://www.rg-rechtsgeschichte.de/rg13

Zitiervorschlag: Rechtsgeschichte Rg 13 (2008)

$\operatorname{Rg} 13_{2008}$

$160-175$

http://dx.doi.org/10.12946/rg13/160-175

\section{Jan Schröder}

Zur Geschichte der juristischen Methodenlehre zwischen 1850 und 1933 


\section{Abstract}

The legal methodology in Germany between 1850 and 1933 has not yet been satisfactorily presented. The author holds that the contemporary concept of law is the key to understanding this matter. In the second half of the 19th century the perception of law as a product of the legislator's or the legal community's will establishes itself against the historical school's theory of persuasion. New concepts of statutes and customary law accord with this concept of law. Statutes are now seen as the expression of the will of the state. Neither the sense of justice nor the legal conviction are, as in the historical school, decisive for customary law, but intention and practice, with a subsequent sense of justice. Finally, judge-made law becomes a source of law, because, on the basis of the voluntaristic concept of law, older subsidiary sources such as natural law and academical law are no longer acknowledged. In this context judge-made law represents only the individual decision, but not a common judicial practice.

Three types of voluntarism can be distinguished: a sociological, which attributes the law creating will to its social preconditions (Jhering, Heck, Ehrlich et al.), an idealistic, which sees it as an attempt to create fair, "right « law (Stammler, Radbruch et al.), and a "normative« type, which restricts itself to the wording of a law and rejects sociological and philosophical explanations (Kelsen). The effects of these differences can be seen, for example, in the theory of interpretation. The so called "subjective interpretation theory is rooted in the sociological, whereas the "objective" is rooted in the idealistic type of voluntarism. 
* Mit Fußnoten versehener Vortrag, den ich am I I. Juli 2007 im MaxPlanck-Institut für europäische Rechtsgeschichte in Frankfurt am Main im Rahmen des Sommerkurses »Europäische Rechtsgeschichte "gehalten habe.

I Siehe etwa die führenden Darstellungen der Privatrechtsgeschichte und der Geschichte des öffentlichen Rechts zum späteren I9. und zum 20. Jahrhundert: FranZ WIEACKER, Privatrechtsgeschichte der Neuzeit unter besonderer Berücksichtigung der deutschen Entwicklung, 2. Aufl., Göttingen I967: 430 ff. (rechtswissenschaftlicher Positivismus), 458 ff. (Übergang zum Gesetzespositivismus), 558 ff. (Entartung des Positivismus und juristischer Naturalismus). Außerdem gibt es einen "soziologischen Positivismus", z. B. bei Bentham, Comte und Mill, 564. Michael Stolleis, Geschichte des öffentlichen Rechts in Deutschland, 2. Band, München I992: "Wissenschafts-« und "Gesetzespositivismus « (28I und öfter) sowie "Naturalismus « (435).

2 Bei Wieacker, Privatgeschichte

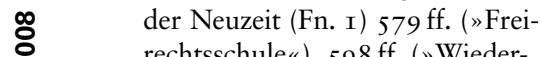
rechtsschule«), 598 ff. (»Wieder-

\section{Zur Geschichte der juristischen Methodenlehre zwischen I 850 und $1933^{*}$}

Ich glaube, niemand wird bestreiten, dass wir bisher über keine brauchbare Darstellung der Rechtsquellen- und Methodenlehre im späten I9. und im frühen 20. Jahrhundert verfügen. Wer heute versucht, sich auf diesem Gebiet zu informieren, begegnet einer Masse verwirrender Schlagworte. Man findet den »Positivismus « in verschiedenen Spielarten. Als Rechtspositivismus oder wissenschaftlichen Positivismus (wobei nicht klar ist, ob damit dasselbe gemeint ist), als "Gesetzespositivismus « oder sogar als "soziologischen Positivismus ", »Empirismus « oder »Naturalismus «. ${ }^{\mathbf{I}}$ Andererseits ist von Phänomenen die Rede, welche offenbar mit einem Positivismus wenig zu tun haben. Von »Freirechtsbewegung " und neuem Naturrecht, ${ }^{2}$ " Antiformalismus $~,{ }^{3}$ "geisteswissenschaftlicher Richtung " im Staatsrecht ${ }^{4}$ usw. Während wir vom naturrechtlichen I8. Jahrhundert und von der historischen Schule im frühen I9. Jahrhundert einigermaßen klare Vorstellungen haben, scheint ein allseits konsentiertes Schlagwort für die Rechtstheorie in den drei, vier Jahrzehnten vor und nach I900 nicht $\mathrm{zu}$ existieren. Das kann daran liegen, dass diese Epoche tatsächlich keine Einheit bildet. Sei es, weil sie eine Übergangsperiode darstellt, sei es, weil seit I 850 überhaupt die Einheit der Rechtstheorie zerfällt und es von da an nur noch einen dauerhaften Pluralismus verschiedener rechtstheoretischer Richtungen gibt. Es kann aber auch daran liegen, dass wir den richtigen Zugang zu dieser Zeit, die uns ja noch sehr nahe liegt, bisher nicht gefunden haben. Vielleicht sehen wir über den vielen widersprüchlichen Details nicht, was die Haupt- und was die Nebenrichtung, was Regel und was Ausnahme ist. Ich meine, dass möglicherweise beides der Fall ist. Einerseits weist die Rechtstheorie zwischen I 850 und I 933 vielleicht wirklich nicht die Einheit auf, die wir in der historischen Schule und im Vernunftrecht zu finden glauben. Andererseits kann man, wenn man sich um die Grundbegriffe bemüht, vielleicht doch zu etwas größerer Klarheit kommen. Und dazu möchte ich im Folgenden ein paar Vorschläge machen.

aufstieg des Naturrechts «, allerdings vor allem für die Zeit nach dem 2. Weltkrieg).

3 Max Weber, Wirtschaft und Gesellschaft (= Grundriß der Sozialökonomik, III. Abteilung), Tübingen I922, 504 ff. (»antiformale Tendenzen in der modernen Rechtsentwicklung «).

4 So im Titel der Untersuchung von KLAus Rennert: Die »geisteswissenschaftliche Richtung « in der
Staatsrechtslehre der Weimarer Republik: Untersuchungen zu Erich Kaufmann, Günther Holstein und Rudolf Smend, Berlin I987; und zum Ganzen STOLleIs, Geschichte des öffentlichen Rechts in Deutschland, 3. Band, München I999, I 53 ff.; DERS, Der Methodenstreit der Weimarer Staatsrechtslehre - ein abgeschlossenes Kapitel der Wissenschaftsgeschichte?, Stuttgart $200 \mathrm{I}$. 
Die Überlegungen, die ich Ihnen vortrage, sind das Ergebnis der Arbeit an der Fortsetzung meiner Geschichte der neuzeitlichen juristischen Methodenlehre, die bis I 850 reicht. ${ }^{5}$ Der Grundgedanke dieser Methodengeschichte ist, dass es keinen Sinn hat, an die Dinge mit modernen Begriffen heranzugehen und sich dafür irgendwelche Belege aus den Quellen zusammenzuklauben. Der einzig mögliche Weg scheint mir vielmehr der zu sein, dass man die zeitgenössischen Begriffe selbst aufsucht und sich von ihnen her das jeweilige Rechtsdenken erschließt. Und da der zentrale Begriff nun einmal der des Rechts selbst ist, muss man sich zunächst einmal um diesen bemühen. Das ist meine feste Überzeugung, dass der jeweilige Rechtsbegriff den Leitfaden zum Verständnis der jeweiligen anderen rechtstheoretischen Phänomene abgibt. Dass man also, wenn man einmal den Rechtsbegriff einer Epoche verstanden hat, auch ein einigermaßen kohärentes Bild ihrer gesamten Rechtstheorie gewinnen kann.

\section{Der Rechtsbegriff zwischen I850 und 1933}

Wie ist nun der Rechtsbegriff zwischen I 850 und I933? Ich kenne keine brauchbare moderne Untersuchung dazu - so seltsam das sein mag, da es sich doch um einen Grundbegriff unserer Wissenschaft handelt. Es gibt also für den wissenschaftlich Fortgeschrittenen wie für den Anfänger keinen anderen Weg, als zunächst einmal geduldig alle zeitgenössischen Belege zu sammeln, die er finden kann. Das habe ich 2004 auch getan, auf meinem Computer eine Datei » Rechtsbegriff « angelegt und monatelang alles Einschlägige aus dem späten 19. und frühen 20. Jahrhundert exzerpiert, was mir in die Hände fiel. Am Ende hatte ich eine Sammlung von 600 Kilobyte und rund 400.000 Zeichen zusammen, etwa 200 Seiten. Das Gesamtbild war aber eher verwirrend. Es mischte sich eine Fülle von Definitionen, die nur schwer auf eine einheitliche Linie zu bringen waren. Altes steht neben Neuem; Definitionen, die versuchen, den ganzen Begriffsumfang zu fassen, stehen neben anderen, die nur bestimmte Gesichtspunkte hervorheben, und je nach dem Zweck des Autors wechselt der Blickwinkel. Bei genauerem Zusehen ergibt sich dann aber allmählich doch ein klareres Bild. Zum einen fällt auf, dass die meisten Definitionen etwas nicht enthalten, was in der frühen Neuzeit noch selbstverständlich

5 JAN SCHRÖDER, Recht als Wissenschaft. Geschichte der juristischen Methode vom Humanismus bis zur historischen Schule (I 500I 850 ), München 200 I. 
6 Weber, Wirtschaft und Gesellschaft (Fn. 3) I7. Das erscheint hier zwar als bloße Nominaldefinition, aber es ist ja nicht zufällig, dass gerade diese gewählt wird.

7 Ludwig ENNECCERUs, Lehrbuch des Bürgerlichen Rechts, I. Band, I. Abteilung: Einleitung, Allgemeiner Teil, Marburg I9I I, $\mathbb{2}$ I II, $5 \mathrm{I}$, meint überhaupt, "die neuere Zeit« (sc. also auch die Gegenwart) führe »das Recht auf den allgemeinen Willen zurück «.

8 Rudolph von Jhering, Geist des römischen Rechts auf den verschiedenen Stufen seiner Entwicklung, 3. Teil, I. Abteilung (I865), 3. Aufl., Leipzig I877, 3 I 8.

9 Peter Harum, Von der Entstehung des Rechts, Innsbruck I863, II.

io Otto Gierke, Deutsches Privatrecht, I. Band, Leipzig I 895, I I6.

i I Karl Binding, Handbuch des Strafrechts, I. Band, Leipzig I 885 , I97; EduARd Hölder, Pandekten. Allgemeine Lehren, Frei-

war, dass nämlich »Recht « nur eine vernünftige, gerechte Ordnung ist. So heißt es etwa (bei Max Weber und verschiedenen anderen Rechtssoziologen), eine Ordnung sei »Recht, wenn sie äußerlich garantiert ist durch die Chance (physischen oder psychischen) Zwanges ... « ${ }^{6}$ Es wird also unausgesprochen vorausgesetzt, dass auch eine unvernünftige und ungerechte Ordnung Recht sein kann. Und zum anderen fällt auf, dass viele Definitionen positiv die Eigenschaft des Rechts als einer Willensäußerung hervorheben. ${ }^{7}$ Ist man darauf einmal aufmerksam geworden, was bei mir allerdings ziemlich lange gedauert hat, dann kann man mühelos eine Fülle weiterer entsprechender Definitionen einsammeln, die das Recht mit dem Willen, also nicht mit der Rechtsüberzeugung, der Vernunft oder der Gerechtigkeit zusammenbringen.

Es scheint sich also in der zweiten Hälfte des I9. Jahrhunderts eine Willenstheorie des Rechts entwickelt zu haben. Das ist etwas Neues im I9. Jahrhundert. Im frühen I9. Jahrhundert findet man, jedenfalls in der historischen Rechtsschule, noch kaum Spuren einer solchen Theorie. Bei Savigny, Puchta usw. ist das Recht nicht ein willkürliches Erzeugnis des Gesetzgebers, sondern ein sinnvolles Produkt des Volksgeists oder der Volksüberzeugung. Aber seit I 850 tritt das Willenselement immer mehr in den Vordergrund. I865 erklärt Rudolf Jhering: »Die Bezeichnung des Rechts im objectiven Sinn als des sallgemeinen Willens gibt in formaler Beziehung das Wesen desselben in einer Weise wieder, wie sie nicht kürzer und treffender gedacht werden kann. ${ }^{8}$ Schon I 863 hatte der Innsbrucker Zivilist Peter Harum das Recht auf ein »über dem Einzelwillen stehendes ... höheres mächtiges Wollen « zurückgeführt. ${ }^{9}$ Nach 1870 verbreitet sich dieser neue voluntaristische Rechtsbegriff mit »bedrohlicher « Schnelligkeit, wie Otto von Gierke schon I 895 besorgt feststellte. ${ }^{\text {Io }}$ Das Recht erscheint jetzt nicht mehr als Rechtsüberzeugung, sondern als »erklärter Gemeinwille « (Karl Binding), »Wille der Gemeinschaft « (Eduard Hölder), »allgemeiner Wille« (Heinrich Dernburg), »unverletzbar selbstherrlich verbindendes Wollen « (Rudolf Stammler), » erklärter Wille der Gemeinschaft “(Philipp Heck) ${ }^{\text {II }}$ oder auch einfach nur als Staatswille (Hans Kelsen). ${ }^{\text {I2 }}$ Andere Autoren stellen nicht auf den Willen, sondern auf das fertige Recht, den Inbegriff von » Normen " ab, fassen aber eben die Norm wieder als Ausdruck eines menschlichen Willens auf. ${ }^{\text {I3 }}$ I9II stellt Ludwig Enneccerus in seinem Lehrbuch des Bürgerlichen Rechts fest: Savigny habe das Recht auf

burg i. Br. I89 I, I8; HEINRICH Dernburg, Pandekten, I. Band, 5. Aufl., Berlin I 896, 43; Rudolf STAMMLER, Theorie der Rechtswissenschaft, Halle I9I I, ro9 (» unverletzbar selbstherrlich verbindendes Wollen «); PHILIPP HEck, Gesetzesauslegung und Interessenjurisprudenz, Tübingen I9I4, I3 (zum Gesetz).

I 2 Hans Kelsen, Hauptprobleme der Staatsrechtslehre (I9II), un- veränderter Nachdruck Tübingen I923, 97.

I 3 Zum Beispiel ERnst Rudolf Bierling, Juristische Prinzipienlehre, I, Freiburg i. Br. und Leipzig I 894, I 9 (Recht enthält Normen, die ein äußeres Verhalten von Menschen regeln und von diesen als Recht anerkannt werden), 29 (eine »Norm ist Ausdruck eines Wollens, das seine Vollziehung von anderen erwartet «). 
die Rechtsüberzeugung gestützt, aber »die neuere Zeit « führe »das Recht auf den allgemeinen Willen zurück, der zwar durch das Rechtsbewusstsein, noch mehr aber durch Gedanken der Zweckmäßigkeit und des Fortschritts bestimmt wird “. ${ }^{\mathbf{I} 4}$ Soweit ich sehe, behauptet sich der voluntaristische Rechtsbegriff jedenfalls bis I930. Allerdings verschwindet in den Definitionen das Willenselement zunehmend hinter farblosen Ausdrücken wie »Ordnung «, "Regelung «, "Norm « usw. und es treten auch alternative Begriffe hervor. Aber noch in der Mitte der zwanziger Jahre bezeichnen verschiedene Autoren den voluntaristischen Rechtsbegriff als jedenfalls herrschend, ${ }^{15}$ und er findet sich nach wie vor bei Juristen unterschiedlichster theoretischer Richtungen: Für Gerhard Anschütz (I927) ist Recht »Ausdruck einer menschlichen Macht, die sich auf den Gemeinwillen stützt «, für Gustav Radbruch (I932) heteronomer "Wille« (jedenfalls soweit es um die richterliche Rechtsanwendung geht) und für Philipp Heck, ebenfalls I932, ein Inbegriff von Geboten, im Gegensatz zur Volksgeistlehre der historischen Schule. ${ }^{\text {I6 }}$

Damit ergibt sich, wie ich meine, für den Rechtsbegriff eine ziemlich klare Entwicklungslinie seit dem I6. Jahrhundert. ${ }^{17} \mathrm{Zu}$ nächst herrscht ein werthaltiger Rechtsbegriff: Recht kann nur sein, was vernünftig, gerecht oder wenigstens zweckmäßig ist. Etwa seit der Mitte des I7. Jahrhunderts verlieren sich die Wertanforderungen. Man betrachtet nun jede Anordnung eines Gesetzgebers als positives Recht. Allerdings wird das Naturrecht noch als Rechtsquelle beibehalten, so dass ich von einem dualistischen Rechtsbegriff (positives Recht und Vernunftrecht) sprechen möchte. Auf der dritten Stufe (historische Rechtsschule) anerkennt man überhaupt nur noch positives Recht, sieht dieses aber nicht als Ausdruck gesetzgeberischer Willkür, sondern als organisches Produkt der Volksüberzeugung an. Unsere Periode tut nun also den letzten Schritt zur Positivierung des Rechts, indem sie das positive Recht auch nicht mehr - wie die historische Schule - idealistisch auflädt, sondern den Willen des Gesetzgebers oder der Rechtsgemeinschaft genügen lässt. Diese Entwicklungslinie und die Voluntarisierung des Rechtsbegriffs seit der zweiten Hälfte des I9. Jahrhunderts scheinen mir außerordentlich wichtig zu sein. Worin ihre politisch-sozialen Gründe liegen, darüber kann man spekulieren. Sicherlich bestehen Zusammenhänge zwischen dem Voluntarismus und dem optimistischen Machbarkeits- und Fortschrittsdenken

I4 Wie o. Fn. 7 .

I 5 Etwa Friedrich DARMSTÄDTER, Recht und Rechtsordnung. Ein Beitrag zur Lehre vom Willen des Gesetzgebers, Berlin-Grunewald I925, I 29; KARL WOLfF, Der Rechtsbegriff, in: Archiv für Rechts- und Sozialphilosophie 20 (I926/27) 279 ff., $288 \mathrm{f}$.

I 6 In der Reihenfolge der Zitate: Gerhard AnschüTz, Diskussionsbemerkung, in: VVDStRL 3
(I927) 47; Gustav Radbruch, Rechtsphilosophie, 3. Aufl., Leipzig I932, 42 f., 83 f.; PHILIPP Heck, Begriffsbildung und Interessenjurisprudenz, Tübingen I932, 54, 72 f., 87 .

I7 Dazu jetzt zusammenfassend JaN SCHröDER, Zur Entwicklung des Rechtsbegriffs in der Neuzeit, in: Gedächtnisschrift für Jörn Eckert, Baden-Baden 2008. 
i 8 Rudolf von Jhering, Geist des römischen Rechts auf den verschiedenen Stufen seiner Entwicklung, I. Band (I 852 ), 4. Aufl., Leipzig I878, 2 I6.

I9 Paul Laband, Das Budgetrecht nach den Bestimmungen der Preussischen Verfassungs-Urkunde unter Berücksichtigung der Verfassung des Norddeutschen Bundes, Berlin I87I, 3; OsKar BüLow, Gesetz und Richteramt, Leipzig I 88 5, 6; GeORg JeLlineK, Gesetz und Verordnung. Staatsrechtliche Untersuchungen auf rechtsgeschichtlicher und rechtsvergleichender Grundlage, Freiburg i. Br. I887, 23 I; AlBERT Haenel, Das Gesetz im formellen und materiellen Sinne (= DERS., Studien zum deutschen Staatsrechte, 2. Band, Heft 2), Leipzig I 888,275 .

20 Binding, Handbuch des Strafrechts (Fn. I I) 200; ENNECCERUS, Lehrbuch des Bürgerlichen Rechts (Fn. 7) $\$ 29$ II, 68; Hölder, Pandekten (Fn. II) 29; Ferdinand Regelsberger, Pandekten, Leipzig I 893, 90; Heck, Gesetzesauslegung und Interessenjurisprudenz (Fn. I I) I3; Kelsen, Hauptprobleme (Fn. I2).

๕ัญे 2i Laband, Budgetrecht (Fn. I9) 3 , 5. Siehe auch DERS., Das Staats-

des späten I9. Jahrhunderts. Umgekehrt bestanden vorher ja auch Zusammenhänge zwischen dem Rechtsbegriff der historischen Schule und der Restauration. Es genügt, wie ich meine, diese $\mathrm{Zu}$ sammenhänge oder Parallelen zu sehen; ob man sie in ein Verhältnis von Ursache und Wirkung bringen kann, erscheint mir zweifelhaft.

Ich gehe damit zum zweiten Teil über und möchte nun im Einzelnen zeigen, wie sich die neue Grundvorstellung vom Recht auch im Detail der Rechtsquellenlehre um I900 wiederfindet.

\section{Der Voluntarismus und die einzelnen Rechtsquellen \\ I. Das Gesetz}

Für die historische Schule war das Gesetz ein Organ des Volksgeistes, d.h. es brachte nicht selbst willkürlich Recht hervor, sondern gab nur der vernünftigen Volksüberzeugung eine Form. Mit dem vordringenden Voluntarismus im späten I9. Jahrhundert verliert sich diese Vorstellung. Das Gesetz wird zur bloßen Willensäußerung des Gesetzgebers. Es ist nun »Wille des Staats" (Jhering), ${ }^{18}$ eine $»$ Äußerung des Staatswillens « (Laband, Bülow,

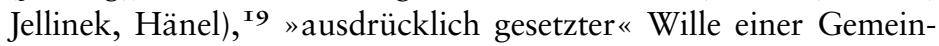
schaft im Gegensatz zum Gewohnheitsrecht (so zum Beispiel bei Binding, Enneccerus, Hölder, Regelsberger, Heck und natürlich auch bei Kelsen). ${ }^{20}$ Der Gesetzesbegriff verändert sich also parallel zum Rechtsbegriff im voluntaristischen Sinne.

Daneben gibt es auch noch andere Gesetzesbegriffe um I900, die zu spezielleren Zwecken gebildet sind. Paul Laband hat bekanntlich die Unterscheidung zwischen dem Gesetz im materiellen und im formellen Sinne eingeführt. ${ }^{2 \mathrm{I}}$ Sie hat aber nur den Zweck, die Kompetenzen von Parlament und Regierung in der konstitutionellen Monarchie voneinander abzugrenzen. Schon fast willkürlich definieren Merkl und Kelsen in ihrer Stufenbaulehre das Gesetz als diejenige Stufe der Normbildung, die zwischen Verfassung und Verordnung steht ${ }^{22}$ usw. Für unsere Fragestellung sind diese Gesetzesbegriffe nicht von Interesse. ${ }^{23}$

\section{Das Gewobnheitsrecht}

Damit komme ich zum Gewohnheitsrecht, dessen Begriff und Voraussetzungen zu den umstrittensten Fragen in unserer Epoche

recht des Deutschen Reiches, 2. Band, 4. Aufl., Tübingen und Leipzig I90I, I, 56. Dazu ErnstWOLFGANG BÖCKENFÖRDE, Gesetz und gesetzgebende Gewalt. Von den Anfängen der deutschen Staatsrechtslehre bis zur Höhe des staatsrechtlichen Positivismus, 2. Aufl., Berlin I98 I, $226 \mathrm{ff}$. 22 Adolf Merkl, Die Lehre vom Wesen der Rechtskraft, entwickelt aus dem Rechtsbegriff, Leipzig und Wien I923, 213, 287; Hans Kelsen, Reine Rechtslehre, Wien I934, 73 ff., 77.

23 Zum Ganzen Jan SCHRÖdER, Gesetzesbegriffe im deutschen Kaiserreich und in der Weimarer Republik, in: BERND-RÜDIGER Kern u. a. (Hrsg.), Humaniora. Festschrift für Adolf Laufs zum 70. Geburtstag, Berlin 2006, $275 \mathrm{ff}$. 
gehören. ${ }^{24}$ Die Fülle der einschlägigen Untersuchungen und die gedankliche Anstrengung, die man dem Problem widmet, stehen in einem seltsamen Missverhältnis zur tatsächlichen Relevanz dieser Rechtsquelle, die um 1900 meistens - und ganz realistisch - als eher unbedeutend eingeschätzt wird. Aber das Gewohnheitsrecht war offenbar ein wichtiger Prüfstein des neuen Rechtsbegriffs. Man empfand wohl, dass gerade hier die Lehre der historischen Schule umgeformt werden musste, und das rechtfertigte vielleicht auch den theoretischen Aufwand.

In der historischen Schule war das Gewohnheitsrecht die zentrale Rechtsquelle. Es wurzelte in der Volksüberzeugung und war im Grunde mit ihr identisch. Die Gewohnheit, die »Übung « als solche ging aus der Volksüberzeugung hervor, sie wurde gar nicht mehr als selbständiges Erfordernis des Gewohnheitsrechts betrachtet, sondern nur als sein Erkenntnismittel. ${ }^{25} \mathrm{Im}$ Voluntarismus des späten I9. Jahrhunderts kehrt sich dieses Verhältnis um: Primär ist nicht mehr die Rechtsüberzeugung (die man auch Rechtsgefühl nennen kann), sondern das tatsächlich geübte Recht. In diesem Sinne schreibt Jhering I877: "Nicht das Rechtsgefühl hat das Recht erzeugt, sondern das Recht das Rechtsgefühl. ${ }^{26}$ Es ist eben der Wille, der das Recht - die Gewohnheit, die tatsächliche Übung - hervorbringt, und nicht die innere "stillwirkende « Überzeugung von der Richtigkeit des Rechts. Denn, noch einmal Jhering (I872), die Überzeugung bildet sich erst, »indem sie handelt«, indem sie »durch dies Handeln ihre Kraft und damit ihren Beruf bewährt, das Leben zu beherrschen ${ }^{27}{ }^{27}$ In diesem Sinne baut man nun die Gewohnheitsrechtslehre der historischen Schule um. Die willensgetragene Gewohnheit wird wieder zum essentiellen Element des Gewohnheitsrechts. ${ }^{28}$ Einige Autoren, wie Georg Jellinek und Ernst Zitelmann, wollen sie überhaupt schon genügen lassen. ${ }^{29}$ Das entspricht zwar nicht der um I900 herrschenden Meinung. Aber auch diese stuft das Merkmal der »Rechtsüberzeugung « gegenüber der Gewohnheit herunter. Die Rechtsüberzeugung muss jetzt nicht mehr eine präexistente sein, wie in der historischen Schule, sondern kann (und wird auch oft) der Gewohnheit nachfolgen. Sie wird (so schreibt Nipperdey I93 I) "praktisch erst nach einer ständigen und dauernden Übung zur Entstehung gelangen «. Oder Radbruch (I9I9): »der tatsächliche Brauch [sc. wird] allmählich mit der Überzeugung der Rechtsnotwendigkeit umkleidet und so zum Gewohnheitsrecht verdichtet. ${ }^{30}$ Wie man die Anforde-

24 Neuere Darstellungen zur Entwicklung der GewohnheitsrechtsTheorie nach I 850 : Alf Ross, Theorie der Rechtsquellen. Ein Beitrag zur Theorie des positiven Rechts auf Grundlage dogmenhistorischer Untersuchungen, Leipzig und Wien I929, 435 f.; HaNs Mokre, Theorie des Gewohnheitsrechts. Problementwicklung und System, Wien I932, 27 ff.; Matthias Frühauf, Zur Legiti- mation von Gewohnheitsrecht im Zivilrecht unter besonderer Berücksichtigung des Richterrechts, Berlin 2006, 44 ff.; JAN SCHRÖDER, Zur Theorie des Gewohnheitsrechts zwischen I 850 und I930, in: Hans-Peter HaferKamp, Tilman Repgen (Hrsg.), Usus modernus pandectarum. Römisches Recht, Deutsches Recht und Naturrecht in der Frühen
Neuzeit. Klaus Luig zum 70. Geburtstag, Köln 2007, 2 I9 ff.

25 Friedrich Carl von Savigny, System des heutigen römischen Rechts, I. Band, Berlin I 840, 35; Georg Friedrich Puchta, Pandekten, 5. Aufl., besorgt von A. RudorfF, Leipzig I850, $\mathbb{I}$ I, I $7 \mathrm{f}$.

26 Rudolf von JHering, Der Zweck im Recht, I. Band, Leipzig I877, XIII.

27 Rudolf von Jhering, Der Kampf um's Recht (I872), Iо. Aufl., Wien I89 I, Iо.

28 Schon in der historischen Schule um I 850 , etwa bei FrIEDRICH Julius Stahl, Die Philosophie des Rechts, 2. Band, I. Abt., 3. Aufl., Heidelberg I 854, 238; GEORG BESELER, Volksrecht und Juristenrecht, Leipzig I 843,88 , auch 303 f., 304 f., 332 .

29 Georg Jellinek, Allgemeine Staatslehre (I900), 2. Aufl., Berlin i 905, 33 I; ERnSt ZitelmanN, Gewohnheitsrecht und Irrtum, in: AcP 66 (I883) 323 ff., 445.

30 Ludwig EnNeccerus, Lehrbuch des Bürgerlichen Rechts, bearb. von Hans Carl Nipperdey, Marburg I93 I, $\mathbb{3} 6$ I 2, I09; Gustav Radbruch, Einführung in die Rechtswissenschaft, 4. Aufl., Leipzig I9I9, 3I. Weitere Nachweise bei SCHRÖDER, Zur Theorie des Gewohnheitsrechts (Fn. 24) 23 I mit Anm. 57. 
3I ERnst Rudolf Bierling, Juristische Prinzipienlehre, 2. Band, Leipzig und Tübingen ז 898, 293, $300 \mathrm{ff} ., 306$.

32 Carl Georg Bruns, Das heutige Römische Recht, in: FranZ vON HoltzendorfF (Hrsg.), Encyklopädie der Rechtswissenschaft in systematischer Verarbeitung, 4. Aufl., Leipzig I88I, 385 ff., 398 f.; Adolf LASSON, System der Rechtsphilosophie, Berlin und Leipzig I 882, 4I7 f.; LABAND, Staatsrecht (Fn. 2I) $\mathbb{5 7}$ III, $69 \mathrm{f}$., s. auch $\mathbb{5} 54$ vor I., 2 mit Anm. I; Binding, Handbuch des Strafrechts (Fn. I I) I98 f. mit Anm. 6, 2io; Rudolf Stammler, Über die Methode der geschichtlichen Rechtswissenschaft, in: Festgabe zu Bernhard Windscheids fünfzigjährigem Doktorjubiläum, Halle I888, I ff., 48 f.; vgl. auch DERS., Lehrbuch der Rechtsphilosophie, 3. Aufl., Berlin und Leipzig I928, I 40 (Gewohnheitsrecht und seine Voraussetzungen gehören zum »Inhalte geschichtlich bedingten Rechts«); Gustav Rümelin, Das Gewohnheitsrecht, in: v. JHERING œ (Hrsg.), Jahrbücher für die DogN. matik des heutigen römischen und

rungen an die Rechtsüberzeugung minimalisiert, zeigt sich vor allem in der sog. "Anerkennungstheorie « Ernst Rudolf Bierlings. Von der Rechtsüberzeugung der historischen Schule bleibt bei ihm und bei seinen Zeitgenossen die »Anerkennung « übrig, die aber nicht mehr sein soll als ein wertindifferentes Hinnehmen der Tatsache, dass ein bestimmter Satz Recht darstellt. Nicht mehr die Überzeugung macht Gewohnheitsrecht, sondern die durch Willen und Macht Einzelner eingeführte Gewohnheit, die dann die übrigen Rechtsgenossen kraft Blankovollmacht so akzeptieren. ${ }^{3 \text { I }}$

Noch radikaler voluntaristisch ist die "staatliche " Theorie des Gewohnheitsrechts, die sogenannte »Gestattungstheorie«. Nach ihr ist Gewohnheitsrecht nicht schon der Wille irgendeiner Gemeinschaft, sondern nur der Wille eines Staates. Es genügt also nicht, dass sich im Volk oder der Gesellschaft irgendwelche Gewohnheiten und Rechtsvorstellungen bilden, sondern der Staat muss ihnen auch zustimmen. Diese Theorie war nicht herrschend, wird aber um I900 von einer Fülle namhafter Juristen vertreten. $\mathrm{Zu}$ ihnen gehören vor I900 Carl Georg Bruns, Adolf Lasson, Karl Binding, Rudolf Stammler, Gustav Rümelin und Paul Laband. ${ }^{32}$ Später kommen vor allem Öffentlichrechtler wie Gerhard Anschütz, Hans Kelsen, Walter Jellinek und Richard Thoma hinzu. ${ }^{33}$ Auch das Reichsgericht geht in seiner Rechtsprechung von der Gestattungstheorie aus. ${ }^{34}$ Sie ist, wie Sie sehen, eine besonders konsequente Umsetzung des Voluntarismus in die Lehre vom Gewohnheitsrecht. Indem sie auch das Gewohnheitsrecht dem Willen des Gesetzgebers unterwirft, schafft sie es als besonderen Rechtsquellentyp eigentlich überhaupt ab.

De facto unterscheidet sich allerdings die »Gestattungstheorie « von der herrschenden Meinung weniger, als es scheint. Darauf möchte ich hier nicht eingehen. Ich meine, es genügt gezeigt zu haben, wie der Voluntarismus auch die Gewohnheitsrechtstheorie durchdringt und sie zu verschiedenen Spielarten umformt.

\section{Das Richterrecht}

Ich wende mich nun der dramatischsten Veränderung um I900 zu, nämlich dem Aufstieg des Richterrechts zur subsidiären Rechtsquelle. In der frühen Neuzeit gab es (wie ich nach wie vor meine) kein Richterrecht in diesem Sinne. Naturrecht, Aequitas und »analogia iuris « standen als ergänzende Rechtsquellen zur

deutschen Privatrechts 27 (I 889) I 53 ff., I 86 ff., 209.

33 Kelsen, Hauptprobleme der Staatsrechtslehre (Fn. I2) IOI; Walter JellineK, Gesetz, Gesetzesanwendung und Zweckmässigkeitserwägungen, Tübingen I9I3, 22 f.; Georg Meyer, Gerhard AnschüTz, Lehrbuch des Deutschen Staatsrechts, 7. Aufl., München und Leipzig I9I9, 234 Anm. I 5 (s. auch Gerhard AnsCHÜTZ in: Preußisches Verwaltungsblatt 22 [I90I] 87 r. Sp. mit Anm. I 8); Richard Thoma, Der Vorbehalt der Legislative und das Prinzip der Gesetzmäßigkeit von Verwaltung und Rechtsprechung, in: Anschütz, Thoma (Hrsg.), Handbuch des deutschen Staatsrechts, 2. Band, Tübingen I932, \76, 22I ff. (230 Anm. 25).

34 RGZ 5, I3O (I34); 4I, 387 (392); II3, 349 (352). 
Verfügung. ${ }^{35}$ Auch die historische Schule kannte und brauchte kein Richterrecht. Wenn Gesetz und Gewohnheitsrecht nicht ausreichten, dann trat als dritte Rechtsquelle das » wissenschaftliche Recht « in die Lücke ein. ${ }^{36}$ Das Recht bildete ja ein aus dem Volksgeist hervorgehendes organisches, vernünftiges Ganzes, aus dem einzelne Lücken ergänzt oder Fehler korrigiert werden konnten. Die Wissenschaft musste diese ergänzenden Regeln nur aufsuchen.

Dieser Ausweg war aber im Voluntarismus nicht mehr gangbar. Wenn das Recht nur noch ein mehr oder weniger zufälliges Willensprodukt ist, dann wird seine innere Vernunft und Vollständigkeit zweifelhaft. Eine wissenschaftliche Ergänzung aus ihm selbst heraus ist nicht immer möglich. Da man aber auch das Naturrecht (schon seit langem) nicht mehr als ergänzende Rechtsquelle anerkannte, gab es jetzt beim Versagen von Gesetz und Gewohnheitsrecht überhaupt kein subsidiäres Recht mehr. Zum ersten Mal in der deutschen Geschichte des gelehrten Rechts stand man also vor einem unvollständigen Rechtsquellensystem. Geradezu zwangsläufig musste man also auf den Gedanken verfallen, dass notfalls eben der Richter selbst das Recht setzt. Wie Sie wissen, kommt dieser Gedanke in den letzten Jahrzehnten des I9. Jahrhunderts gleichzeitig mit der Ausbildung des voluntaristischen Rechtsbegriffs auf. Franz Adickes möchte I 872 die »subjektive Vernunft « des Richters wenigstens als subsidiäre Rechtsquelle einsetzen, Oskar Bülow fordert I 885 , man müsse sich »zu einem eigenartigen, neben dem Gesetzesrecht stehenden ... richterlichen Recht bekennen « und Gustav Rümelin hebt I89I die Bedeutung persönlicher »Werturteile und Willensentscheidungen « des Richters hervor. ${ }^{37}$ Die sogenannte »Freirechtsbewegung «, beginnend mit den Schriften von Eugen Ehrlich (I903), Ernst Stampe (I905) und Gnaeus Flavius = Hermann Kantorowicz (I906), ${ }^{38}$ leistet dann dem Gedanken der richterlichen Rechtsschöpfung weiter Vorschub und setzt ihn, wie ich meine, durch.

Allerdings mit zwei Einschränkungen. Die erste ist, dass einige besonders exzentrische freirechtliche Vorstellungen nicht übernommen werden. Die Idee, dass das Gesetz »nicht weniger Lücken hat als Worte «, 39 dass es nur so weit reicht wie sein klarer Wortlaut (Kantorowicz, Stampe, Fuchs), hat sich nicht durchgesetzt. Wir und schon die meisten Juristen in den ersten Jahrzehnten nach I 900 geben dem Gesetz durch Auslegung und Analogie einen sehr viel größeren Spielraum. Aber es bleibt ein Kernbereich von Über-
35 Dazu Schröder, Recht als Wissenschaft (Fn. 5) I 5 ff. ("aequitas" in der frühesten Neuzeit), I08 ff. (Naturrecht und "analogia iuris « im I7./I 8. Jahrhundert).

36 Dazu SchröDER, Recht als Wissenschaft (Fn. 5) I98 ff.

37 In der Reihenfolge der Zitate:

Franz Adickes, Zur Lehre von den Rechtsquellen, insbesondere über die Vernunft und die Natur der Sache als Rechtsquellen und über das Gewohnheitsrecht, Kassel und Göttingen I872, 67 und öfter; Oskar BüLOw, Gesetz und Richteramt, Leipzig I885, 2, 6; Gustav Rümelin, Werturteile und Willensentscheidungen im Civilrecht, Freiburg i. Br. I89 I, 22 und öfter.

38 Eugen Ehrlich, Freie Rechtsfindung und freie Rechtswissenschaft, I903; ERnSt STAMPE, Unsere Rechts- und Begriffsbil- dung, Greifswald I907, I-9 (Sammlung von Aufsätzen aus DJZ I905, Sp. 4I 7 ff., 7I 3 ff., IOI 7 ff.); GNAEUs Flavius, Der Kampf um die Rechtswissenschaft, Heidelberg I906.

39 So Gnaeus flavius, Der Kampf um die Rechtswissenschaft (Fn. 38) I 5 . 
40 Philipp Heck, Gesetzesauslegung (Fn. II) 248 f.; MAX RüMELIN, Erlebte Wandlungen in Wissenschaft und Lehre, Tübingen I930, 33.

4I Gustav Radbruch, Rechtswissenschaft als Rechtsschöpfung, (I906), in: DERs., Gesamtausgabe, Band I, Rechtsphilosophie I, bearb. von ARTHur KaUfManN, Heidelberg I987, 409 ff., 4I7; Kelsen, Reine Rechtslehre (Fn. 22) 98, der allerdings die Lückenvorstellung kritisiert.

42 Walter Schönfeld, Der Traum des positiven Rechts, in: AcP I35 (I932) I ff., 6I; ERICH KAUFMANN, Die Gleichheit vor dem Gesetz im Sinne des Artikels I09 der Reichsverfassung, in: VVDStRL 3 (I927) 2 ff., 2I; GÜNTHER HolsteIn, Die Grundlagen des evangelischen Kirchenrechts, Tübingen I928, 345 .

43 Josef Unger, Der Kampf um die Rechtswissenschaft, in: DJZ I906, Sp. 78 I ff., 784; ERNST RUdOLF BIERLING, Juristische Prinzipienlehre, 4. Band, Tübingen I9II, 397; Paul Oertmann, Gesetzeszwang und Richterfreiheit, Leipzig I909, 24 ff.; ENNECCERUS, NipPERDEY, Lehrbuch des Bürgerli-

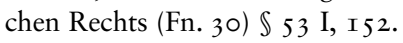
44 S. außer den im Vorigen (Fn. 3942) zitierten noch: STAMPE, Rechts- und Begriffsbildung (Fn. 38) 23 f.; Max RumpF, Gesetz und Richter. Versuch einer Methodik der Rechtsanwendung, Berlin I906, I03, LORENZ BRÜTT, Die Kunst der Rechtsanwendung, Berlin I907, I 80 f.; GÉZA KIss, Gesetzesauslegung und »ungeträge zur Theorie der Rechtsquel-

einstimmung mit den Freirechtlern, nämlich der, dass Lücken im Recht (mögen es nun viele oder wenige sein) prinzipiell unvermeidlich sind und dass sie nur der Richter schließen kann. Darin sind fast alle Autoren des frühen 20. Jahrhunderts einig: Interessenjuristen wie Philipp Heck und Max Rümelin, ${ }^{40}$ Neukantianer wie Gustav Radbruch und Hans Kelsen, ${ }^{4 \mathbf{I}}$ Neuhegelianer wie Walter Schönfeld, Erich Kaufmann und Günther Holstein ${ }^{42}$ und eine breite Gruppe von Autoren, für die mir keine Sammelbezeichnung mehr einfällt, wie Josef Unger, Ernst Rudolf Bierling, Paul Oertmann, Hans Carl Nipperdey ${ }^{43}$ und andere. Die Unentbehrlichkeit der richterlichen Rechtsschöpfung als solche ist im frühen 20. Jahrhundert fast unumstritten (und das macht es auch so schwierig, die Freirechtler von den Nichtfreirechtlern überhaupt noch zu unterscheiden).

Die zweite Einschränkung: Mit »Richterrecht « meine ich nicht eine ständige Gerichtspraxis. Sie ist als solche keine Rechtsquelle, weil unserem kodifizierten Rechtssystem ein Präjudizienrecht fremd ist. Eine ständige Gerichtspraxis kann nur als Gewohnheitsrecht zur Rechtsquelle werden; das war auch um I900 die ganz herrschende Meinung. »Richterrecht « bedeutet also nur, dass der Richter für den jeweiligen Einzelfall ohne präjudizielle Wirkung Recht setzt, wenn er den in Gesetz und Gewohnheitsrecht nicht vorhandenen Obersatz seiner Entscheidung selbst findet. ${ }^{44}$ In diesem Sinne war das »Richterrecht « als Rechtsquelle nach I900 kaum umstritten. Letztlich ist das, um es noch einmal zu sagen, eine Konsequenz des voluntaristischen Rechtsbegriffs. Es ist der Ausweg aus dem Dilemma, dass man einerseits kein generelles subsidiäres Recht neben dem Gesetzes- und Gewohnheitsrecht mehr akzeptierte, andererseits aber auch nicht das englisch-amerikanische Präjudiziensystem. ${ }^{45}$

Zusammenfassend darf man also feststellen: Der im späten I9. Jahrhundert aufkommende rechtstheoretische Voluntarismus wandelt die Rechtsquellenlehre völlig um. Das Gesetz wird zum Willen des Gesetzgebers. Das Gewohnheitsrecht wird zur willentlich erzeugten, von der Rechtsgemeinschaft dann hingenommenen Übung. Und da der Voluntarismus keine subsidiäre Rechtsquelle, wie Naturrecht oder wissenschaftliches Recht, mehr kennt, muss nun unter gewissen Umständen auch der Richterspruch Recht schaffen, wenn auch nur für den jeweiligen Einzelfall.

len, in: JHERING (Hrsg.), Jahrbücher für die Dogmatik des bürgerlichen Rechts 58 (I9II) 4I3 ff., 466 f.; CARL SCHMITT, Gesetz und Urteil (I9I2), 2. Aufl., München I969, 97; Alfred MANigK, Savigny und der Modernismus im Recht, Berlin I9I4, I 22 (»ius concretum «); HeINRICH LeHMANN, Allgemeiner Teil des Bürgerlichen Gesetzbuchs, 3. Aufl., Berlin und Leipzig I928, 26;
ERnst Fuchs, Was will die Freirechtsschule?, Rudolstadt I929, I6.

45 Zum Ganzen: JaN SCHRÖDER, »Richterrecht « und Rechtsbegriff im frühen 20. Jahrhundert, in: Klaus Peter Berger u. a. (Hrsg.), Zivil- und Wirtschaftsrecht im europäischen und globalen Kontext. Festschrift für Norbert Horn zum 70. Geburtstag, Berlin 2006, I $255 \mathrm{ff}$. 


\section{Die Spielarten des voluntaristischen Rechtsbegriffs}

Dies alles klingt nun schon fast zu harmonisch, vielleicht sogar etwas gewaltsam vereinfachend. Sie werden fragen: Wo bleibt die um I900 wieder erstehende Rechtsphilosophie, die geisteswissenschaftliche Richtung im Staatsrecht, überhaupt der Neo-Idealismus der I92oer Jahre? Und wie ist z. B. die »soziologische « Jurisprudenz im Voluntarismus zu verorten? Die Fragen sind berechtigt. Ich meine, man muss sehen, dass der Voluntarismus nur das gemeinsame Dach für verschiedene Spielarten des Rechtsbegriffs ist, von denen ich drei unterscheiden möchte: eine soziologische, eine idealistische und eine normative Variante.

\section{Die soziologische Variante}

Als Begründung der soziologischen Rechtstheorie gilt Rudolf von Jherings zweibändiges Buch »Der Zweck im Recht « (I877I 883). Jhering stellt hier neben seinen voluntaristischen Rechtsbegriff einen zweiten materiellen, wonach das Recht »die Sicherung der Lebensbedingungen der Gesellschaft in Form des Zwanges « ${ }^{\mathbf{6}}$ ist. Es dient also bestimmten Zwecken, wie überhaupt der Zweck nach dem Motto des Buches »der Schöpfer des ganzen Rechts « ist. Welcher der zahlreichen und einander bekämpfenden gesellschaftlichen Zwecke sich schließlich durchsetzt, bestimmt das »Machtverhältniss der sich gegenüberstehenden Kräfte«. Das mächtigere Interesse siegt, oder es kommt zu einem Kompromiss, der dann die Diagonale im »Parallelogramm der Kräfte « ausmacht. ${ }^{47}$ Eine praktische Nutzanwendung für die juristische Methodenlehre bekommt diese Theorie durch Philipp Hecks »Interessenjurisprudenz«. Nach Heck ist auch »jede Fallentscheidung als eine Abgrenzung einander gegenüberstehender Interessen aufzufassen und durch eine Abwägung dieser Interessen nach Werturteilen und Wertideen zu gewinnen $«{ }^{48}$ Man erhält diese Werturteile, indem man hinter das Gesetz (oder Gewohnheitsrecht) auf die dafür ursächlichen Interessen zurückgreift. Hecks Lehre war für das Zivilrecht konzipiert und gewann dort zunehmend an Einfluss, vor allem bei den anderen Vertretern der »Tübinger Schule «, Max Rümelin und Heinrich Stoll, ${ }^{49}$ weiterhin auch bei Eugen Ehrlich und Paul Oertmann. Sie fand aber auch Anhänger in den anderen Rechtsgebieten, etwa im Strafrecht bei August Hegler und im

46 JHERING, Zweck im Recht, I (Fn. 26) 434.

47 JHERING, Kampf um's Recht (Fn. 27) 7 .

48 Philipp Heck, Das Problem der Rechtsgewinnung (I9I 2), 2. Aufl., Tübingen I932, 30.

49 S. etwa Hans Stoll, Rechtsstaatsidee und Privatrechtslehre, in: AcP 76 (I926) I 34 ff., I 57, I60; MAX RÜMELIN, Gesetz, Rechtsprechung und Volksbetäti- gung auf dem Gebiet des Privatrechts. Erster Vortrag, in: AcP I 22 [= n. F. 2] (I924) I45-I72; zweiter und dritter Vortrag, in: AcP I 22 (1924) 265 ff., 296: Rechtsordnung als »Fixierung des Kräfteausgleichs «. 
öffentlichen Recht bei Heinrich Triepel. Die ganze Richtung ist dadurch charakterisiert, dass sie das Recht oder den rechtserzeugenden Willen aus seinen »natürlichen«, sozialen Ursachen herleitet.

\section{Die idealistische Variante}

Im Gegensatz dazu bezieht die »idealistische Variante « den rechtserzeugenden Willen nicht auf die realen gesellschaftlichen Kräfte, die ihn hervorbringen, sondern auf bestimmte höhere, ideale Anforderungen an das Recht, etwa Gerechtigkeit, Vernünftigkeit, Zweckmäßigkeit oder ganz allgemein »Richtigkeit «. So ist für Rudolf Stammler positives Recht zwar jedes »unverletzbar selbstherrlich verbindende Wollen ${ }^{5 \circ}{ }^{\circ}$ Das heißt, Recht ist an sich jede Norm beliebigen Inhalts, wenn sie sich nur an einen anderen wendet (verbindend), ihre Geltung nicht vom Willen des Adressaten abhängig macht, sondern notfalls durch Zwang durchsetzt (selbstherrlich) und auch den Normsetzer bindet (unverletzbar). Im Prinzip ist also auch Stammler ein Voluntarist. Aber, so lehrt er, die »Richtigkeit « dieses positiven Rechts ist von seinem Begriff und der Methode seiner Anwendung nicht völlig zu trennen. Das positive Recht ist jedenfalls »ein Versuch demnächst das Richtige zu treffen «, ein »Zwangsversuch zum Richtigen $« .{ }^{5 \text { I }}$ In Abgrenzung zur soziologischen Theorie könnte man sagen: Nach Stammlers philosophischer Lehre ist der rechtserzeugende Wille nicht nur ein Produkt von Interessenkämpfen, sondern vor allem ein Bemühen, »richtiges Recht « zu erzeugen, und ein richterliches Urteil nicht vornehmlich eine Lösung von Interessenkonflikten, sondern ein Versuch, den Fall »richtig « zu entscheiden (soweit die Bindung des Richters an das Gesetz das zulässt). Ähnliche Vorstellungen finden sich auch bei anderen Rechtsphilosophen der Zeit. Gustav Radbruch nennt das Recht »ein Wollen und Sollen", heteronomen $»$ Willen $«{ }^{52}$ Zugleich ist es aber auch »die Wirklichkeit, die den Sinn hat, dem Rechtswerte, der Rechtsidee zu dienen «. ${ }^{53}$ Auch Neuhegelianer wie Josef Kohler und Julius Binder sehen im Recht zwar den geschichtlichen Ausdruck eines Willens. Dieser soll aber nicht beliebig und zufällig sein, sondern »jeder Kultur das ihr zukommende Recht zu(zu)teilen « (Kohler), es soll in ihm »die Rechtsidee funktionier(en)t « (Binder). ${ }^{54}$ In dieser Weise verbinden sich bei den meisten Autoren Voluntarismus und Idealismus: Recht ist

50 Stammler, Theorie der Rechtswissenschaft (Fn. II) IO9.

5I Stammler. Theorie der Rechtswissenschaft (Fn. II) 747, 649 und öfter.

52 RaDbruCH, Einführung in die Rechtswissenschaft (Fn. 3o) 2 f.

53 RADBRUCH, Rechtsphilosophie (Fn. I6) 29.

54 Josef KoHLER, Lehrbuch der Rechtsphilosophie, Berlin und Leipzig I909, 2, aber auch DERS.,
Lehrbuch des Bürgerlichen Rechts, I. Band: Allgemeiner Teil, Berlin I904, 8 ("geschichtlich gegebene Regelung «); JuLIUS Binder, Philosophie des Rechts, Berlin 1925 , 238 , aber auch 9II (Rechtsnorm ist »Ausdruck eines Willens«). 
zwar schon jede Willensäußerung des Gesetzgebers, aber sie strebt Gerechtigkeit oder Richtigkeit jedenfalls an. ${ }^{55}$

\section{Die normative Variante}

Die dritte Spielart des Voluntarismus, Hans Kelsens »Reine Rechtslehre «, »reinigt « dagegen den Rechtswillen von seinen soziologischen und philosophischen Bezügen. Das Recht ist »Wille des Staates " ${ }^{56}$ aber nicht in einem natürlich-psychologischen, sondern in einem rein »juristischen « Sinne. Es handelt sich bei dem Staatswillen um eine nur »zum Zwecke der Zurechnung vollzogene(n) Konstruktion «. ${ }^{57}$ Für die Rechtswissenschaft ist das Recht kein natürliches, seelisches oder gesellschaftliches Phänomen, kein Sein, sondern nur ein Sollen, ein Inbegriff von Normen. ${ }^{58}$ Es hat weder etwas zu tun mit den realen, psychischsozialen Ursachen der Normen, noch steht es in Verbindung mit Philosophie, richtigem Recht und Gerechtigkeit. Die Rechtswissenschaft wird so "von allen ihr fremden Elementen « befreit, ${ }^{59}$ insbesondere von Ideologien, die unter dem Vorwand der »Gerechtigkeit « doch nur mit den Mitteln des Rechts irgendwelche politischen Interessen durchsetzen wollen. ${ }^{60}$

Vergleicht man abschließend die drei Spielarten des Voluntarismus, dann kann man sagen: Nach der soziologischen Theorie ist das Recht ein Produkt von Interessenkämpfen, nach der idealistischen ein Versuch, die Rechtsidee zu verwirklichen, nach der reinen Rechtslehre nichts anderes als ein Inbegriff von Normen, ein reines Sollen. Immer aber ist es auch eine Willensäußerung des Gesetzgebers. Und die Fallentscheidung ist nach der soziologischen Theorie eine Abgrenzung von Interessenkonflikten, nach der idealistischen eine Bemühung um die »richtige«, gerechte Lösung (immer im Rahmen der gesetzlichen Vorgaben), nach der reinen Rechtslehre nur eine Anwendung von Normen oder, wo diese versagen, eine freie Normsetzung durch den Richter.

Worin liegt nun die methodengeschichtliche Nutzanwendung der Einsicht in die verschiedenen Spielarten des Voluntarismus? Ich meine, dass sie jedenfalls bei der Gesetzesauslegung eine Rolle spielen. Denn es ist ja offensichtlich, dass die Auslegung anders ausfallen muss, wenn man sich unter dem Recht nur ein Erzeugnis gesellschaftlicher Ursachen vorstellt, als wenn man es als »Zwangsversuch zum Richtigen « betrachtet, und wieder anders, wenn man

$55 \mathrm{Zu}$ nennen sind z. B. noch BiNDING, Handbuch des Strafrechts (Fn. I I) I97 (Recht als erklärter Gemeinwille), 455 (Gesetzgeber soll »brauchbare Lebensregeln « schaffen); ADOLF WACH, Handbuch des deutschen Zivilprozeßrechts, I, Leipzig I 885, 260 (Recht ist seiner Natur nach Willenserklärung), 257 (Recht ist seiner Idee nach »das vernünftige Wesen des sich zur allgemeinen Norm eig- nenden Willens «); BRÜTT, Kunst der Rechtsanwendung (Fn. 44) 32 (Rechtsnorm als "sozialer Imperativ«), I I 2 f. (folgt Stammlers Rechtsbegriff), 29 (gerechtes Recht als Wunschrecht, Ideal), I I 8, I34, I3 8 (es gibt ein " prinzipiell richtiges « Recht); HaNs REICHEL, Gesetz und Richterspruch. Zur Orientierung über Rechtsquellen- und Rechtsanwendungslehre der Gegenwart, Zürich
I9I 5, 68, 70, 77 (Gesetz ist "allgemeiner Wille «, aber »seiner Idee nach nicht Willkürprodukt, sondern Vernunfterzeugnis «); ARThur Baumgarten, Die Wissenschaft vom Recht und ihre Methode. I. Die theoretische Grundlegung, Tübingen I920, I62 f. (Recht besteht aus "Befehlen ", aber letztlich ist »die ideale und doch positive Lebensordnung ... das Recht «).

56 Kelsen, Hauptprobleme der Staatsrechtslehre (Fn. I2) 97.

57 Kelsen, Hauptprobleme der Staatsrechtslehre (Fn. I 2) I 84.

58 Kelsen, Reine Rechtslehre (Fn. 22) $6 \mathrm{f} ., 20 \mathrm{f}$.

59 Kelsen, Reine Rechtslehre (Fn. 22) I.

60 Kelsen, Reine Rechtslehre (Fn. 22) V-VII, I7. 
es als von allen Zwecken und Idealen isolierte »reine « Norm sieht. Dazu also noch einige Überlegungen.

\section{Die Spielarten des Voluntarismus und die Gesetzesauslegung}

In der Gesetzesauslegung stehen sich bekanntlich seit langem die sogenannte »objektive " und »subjektive « Theorie gegenüber. Nach der objektiven Theorie kommt es darauf an, was der Gesetz zur Anwendungszeit vernünftigerweise sagen sollte. Nach der subjektiven darauf, was der historische Gesetzgeber sagen wollte.

Die »objektive « Theorie ist, wie Sie wissen, I 88 5-1 886 in drei voneinander unabhängigen Schriften von Binding, Wach und Kohler begründet worden. ${ }^{6 \mathrm{I}}$ Das Gesetz ist so zu interpretieren, wie es nach dem Verständnis der Anwendungszeit richtig erscheint, so, wie es der jeweilige »vernünftig auslegende Volksgeist « (Binding) ${ }^{62}$ versteht. Ich halte diese Lehre für ein charakteristisches Produkt dessen, was ich »idealistischen Voluntarismus " genannt habe. Der Zusammenhang ist bei ihren drei Begründern ganz offensichtlich. Von Kohlers Idee, dass das Recht der jeweiligen zeitgenössischen Kultur entsprechen soll, und damit möglichst auch seine Auslegung, war schon die Rede. ${ }^{63}$ Nach Binding ist das Recht »erklärter Gemeinwille«, aber kein wertneutrales Produkt gesellschaftlicher Verhältnisse, sondern ein Versuch, »brauchbare Lebensregeln « zu schaffen. ${ }^{64}$ Ganz deutlich wird der Zusammenhang bei Adolf Wach, für den das Recht seiner Natur nach eine »Willenserklärung «, »seiner Idee nach « aber »das vernünftige Wesen des sich zur allgemeinen Norm eignenden Willens « darstellt, ${ }^{65}$ » und das Gesetz so auszulegen ist, wie es seinem erkennbaren Zweck und den Forderungen der Gerechtigkeit am meisten entspricht «. ${ }^{66}$ Die Verbindung zwischen idealistischem Rechtsbegriff und »objektiver " Theorie setzt sich fort bei Rudolf Stammler. Nach ihm zielt jedes Recht darauf ab, »richtiges « Recht zu sein und deshalb ist im Zweifel einem Rechtssatz auch der Sinn zuzusprechen, der ihn »als grundsätzlich richtiges Mittel im Zusammenhang der gerade gegebenen Zwecke erscheinen läßt « ${ }^{67} \mathrm{Nach}$ Gustav Radbruch soll das Recht »der Rechtsidee dienen « und dementsprechend ist für ihn auch die Auslegung »eine Gegebenheit, die den Sinn hat, die Rechtsidee zu verwirklichen ${ }^{68}$ und nicht

6I Binding, Handbuch des Strafrechts (Fn. II) 454 ff.; WACH, Handbuch (Fn. 55) 256 ff.; JoseF KoHLER, Über die Interpretation von Gesetzen, in: Zeitschrift für das Privat- und Öffentliche Recht der Gegenwart, hrsg. von C. S. GRÜNHUT I 3 (I886) I ff., 3. 62 Binding, Handbuch des Strafrechts (Fn. II) 456.

63 S. o. zu Fn. 54.

64 S. o. Fn. 55.
65 S. o. Fn. 55

66 WACH, Handbuch des deutschen Zivilprozeßrechts (Fn. 55) $257 \mathrm{f}$. 67 STAMmLER, Theorie der Rechtswissenschaft (Fn. II) 620.

68 RADBRUCH, Rechtsphilosophie (Fn. I6) II6.

Zur Geschichte der juristischen Methodenlehre zwischen I 850 und 1933 
den historischen Willen des Gesetzgebers. Die Belege lassen sich vermehren (Lorenz Brütt, Hans Reichel und Arthur Baumgarten). ${ }^{69}$

Dagegen ist die »subjektive « oder »historische « Auslegungslehre verbunden mit der "soziologischen "Variante der Willenstheorie, wie sie von Jhering, Heck und der Tübinger Schule und einer Reihe anderer Juristen vertreten wird. Wenn das Recht ein kausales Produkt gesellschaftlicher Einflüsse, Interessen und Zwecksetzungen darstellt, dann ist eben auch bei seiner Auslegung auf diese Ursachen zurückzugehen. Philipp Heck hat unermüdlich für eine solche »historische Interessenforschung « geworben und ihr in seiner Monographie »Gesetzesauslegung und Interessenjurisprudenz « von I9 I 4 eine eindrucksvolle Grundlegung gegeben. Der Gesetzesbefehl ist auf seine Ursachen zurückzuführen, d. h. der Interpret muss historisch erforschen, welche Interessen der Befehlsoder Gesetzgeber verfolgt, bzw. welchem von verschiedenen widerstreitenden Interessen er den Vorzug gegeben hat. ${ }^{7 \circ}$ Ebenso kann nach Stoll die Arbeit des Richters (Auslegers) »nur in der Erforschung und Befolgung der Interessenwertungen des historischen Gesetzgebers bestehen ${ }^{{ }^{7 \mathrm{I}}}$ Denselben Standpunkt nimmt Hecks und Stolls Tübinger Kollege Max Rümelin ein, ${ }^{72}$ Ehrlich und Bierling stehen ihm nahe. ${ }^{73}$

Und schließlich ist es auch klar, dass die dritte Spielart des Voluntarismus, Kelsens »normative « Theorie, weder eine objektive (idealistische) noch eine subjektive (soziologische) Interpretation akzeptieren kann. In der »Reinen Rechtslehre « muss die Gesetzesinterpretation beim klaren Normtext stehen und von soziologischen und philosophischen Erwägungen frei bleiben. Wenn ein Gesetz mehrdeutig oder widersprüchlich ist oder sich der sprachliche Ausdruck und der Wille des Gesetzgebers möglicherweise nicht decken, dann trifft die Rechtsnorm selbst eben keine eindeutige Entscheidung und überlässt die Normerzeugung einer anderen Instanz, etwa dem Richter. Innerhalb des vorgegebenen Rahmens ist jede Entscheidung richtig. ${ }^{74}$ Eine »objektive « oder "subjektive " Methode der Interpretation, die diesen Rahmen ausfüllen könnte, gibt es nicht. ${ }^{75}$ Die juristische Interpretation macht vielmehr schon halt, bevor sie zu diesen Methoden und zu den wirklichen Interpretationsproblemen kommt. Geht sie darüber hinaus, dann trifft sie keine »rein « juristische Entscheidung mehr, sondern eine politische. ${ }^{76}$

69 BRÜTT, Kunst der Rechtsanwendung (Fn. 44) I 29 (Lückenfüllung - auch Auslegung? - soll sich nach dem richten, was »die Kulturentwicklung des Volks nach Möglichkeit fördert «); REICHEL, Gesetz und Richterspruch (Fn. 55) 78 (Auslegung nach »sozialer Angemessenheit «); Baumgarten, Die Wissenschaft vom Recht (Fn. 55) 296 (Auslegung nach dem, was »für die Interessen der Allgemein- heit am förderlichsten ist «) Weitere Nachweise bei JAN SCHRÖDER, Rechtsbegriff und Auslegungsgrundsätze im frühen 20. Jahrhundert, in: UlRICH WACKERBARTH u. a. (Hrsg.), Festschrift für Ulrich Eisenhardt zum 70. Geburtstag, München 2007, I 25 ff., I 30 ff..

70 Heck, Gesetzesauslegung und Interessenjurisprudenz (Fn. I I) 45, 49 ff., 59 ff., 89 ff.
7I Stoll, Rechtsstaatslehre und Privatrechtsidee (Fn. 49) I67 f.

72 RÜmelin, Gesetz, Rechtsprechung und Volksbetätigung (Fn. 49) 268 ff.; auch schon DERs., Das neue schweizerische Zivilgesetzbuch und seine Bedeutung für uns, Tübingen I908, $37 \mathrm{f}$.

73 Eugen Ehrlich, Die richterliche Rechtsfindung aufgrund des Rechtssatzes (I9I7), in: DERS., Recht und Leben, hrsg. von Manfred Rehbinder, Berlin I967, 203 ff., 2I9; BIERLING, Juristische Prinzipienlehre, IV (Fn. 43) $230 \mathrm{f}$.

74 Kelsen, Reine Rechtslehre (Fn. 22) 94.

75 Kelsen, Reine Rechtslehre (Fn. 22) $96 \mathrm{f}$.

76 Kelsen, Reine Rechtslehre (Fn. 22) 98. 
Letztlich ist also der Streit zwischen objektiver und subjektiver (und normativer) Auslegungstheorie gar nicht mit Argumenten zu entscheiden. Es handelt sich um zwei verschiedene juristische Weltanschauungen, eine idealistische und eine soziologische (und eine »rein « juristische). Eine wissenschaftlich richtige Entscheidung zwischen diesen Weltanschauungen gibt es nach meiner Überzeugung nicht und darum ist der Streit um die Auslegungsmethoden unlösbar, also systematisch im Grunde überflüssig. Nützlich scheint mir aber die historische Einsicht zu sein, die uns diese Zusammenhänge erkennen lässt. Die Geschichte der juristischen Methodenlehre führt hier, wie ich meine, weiter als die unhistorische moderne systematische Methodologie.

\section{Schluss: Zur Begriffsbildung in der Methodengeschichte des späten I9. und frühen 20. Jabrhunderts}

Lassen Sie mich zum Schluss wenigstens noch ein paar Sätze dazu sagen, wie man nun diese Rechtstheorie der drei, vier Jahrzehnte vor und nach 1900 charakterisieren könnte. Von den vielen Positivismen scheint mir "wissenschaftlicher Positivismus" unpassend, weil er die Methode der historischen Schule und ihrer Epigonen bezeichnen soll. Sie ist aber nicht positivistisch, sondern idealistisch. 77 »Gesetzespositivismus « trifft nicht zu, weil die meisten Autoren außer dem Gesetz auch das Gewohnheitsrecht und den Richterspruch als Rechtsquelle kennen. Die Anzahl der echten Gesetzespositivisten ist klein, und mit Recht hat Karl Kroeschell schon vor zwanzig Jahren vermutet, dass es einen Gesetzespositivismus als besondere wissenschaftliche Theorie nie gegeben habe. ${ }^{78}$ "Soziologischer Positivismus" wiederum ist viel zu eng, weil es nur einen kleinen Teil der Phänomene abdeckt. Es bleibt »Rechtspositivismus «. Wenn man darunter versteht, dass als Recht nur erfahrbare Gegebenheiten anerkannt und nicht (wie in der historischen Schule) idealistisch aufgeladen werden, dann ist gegen diese Bezeichnung für unsere Epoche an sich nichts einzuwenden. Sie könnte allerdings die Vorstellung suggerieren, das Recht sei hier als völlig wertfrei und gerechtigkeitsblind angesehen worden, und würde damit auch in die Irre führen. Denn der Gerechtigkeitsgedanke lässt sich aus dem Recht offenbar nicht vertreiben - in unserer Epoche verschafft er sich Zugang durch die idealistische

77 JOACHIM RÜCKERT, Idealismus, Jurisprudenz und Philosophie bei Friedrich Carl von Savigny, Ebelsbach 1983,240 und passim. Zusammenfassend SCHRÖDER, Recht als Wissenschaft (Fn. 5) I93.

78 Karl Kroeschell, Deutsche Rechtsgeschichte 3, Opladen I989, 266. S. auch JAN SCHRÖDER, Gab es im deutschen Kaiserreich einen Gesetzespositivismus?, in: WOLFGANG BaUmanN u. a.
(Hrsg.), Gesetz, Recht, Rechtsgeschichte. Festschrift für Gerhard Otte, Berlin 2005, 57I ff. 
Variante der Willenstheorie und die »objektive« Auslegungslehre. Aus diesem Grund ist leider auch der von mir verwendete Begriff »Voluntarismus « nicht restlos geeignet. Mit anderen Worten: Ein passendes Schlagwort fehlt uns noch und vielleicht gibt es auch gar keins.

Jan Schröder 